\title{
BMJ open Comparing the performance characteristics of CSF-TRUST and CSF-VDRL for syphilis: a cross- sectional study
}

\author{
Weiming Gu, ${ }^{1}$ Yang Yang, ${ }^{1}$ Lei Wu, ${ }^{1}$ Sheng Yang, ${ }^{1}$ Lai-King $\mathrm{Ng}^{2}$
}

To cite: Gu W, Yang Y, Wu L, et al. Comparing the performance characteristics of CSF-TRUST and CSF-VDRL for syphilis: a cross-sectional study. BMJ Open 2013;3:e002204. doi:10.1136/bmjopen-2012002204

- Prepublication history for this paper are available online. To view these files please visit the journal online (http://dx.doi.org/10.1136/ bmjopen-2012-002204).

Received 10 October 2012 Revised 16 January 2013 Accepted 17 January 2013

This final article is available for use under the terms of the Creative Commons Attribution Non-Commercial 2.0 Licence; see http://bmjopen.bmj.com

\begin{abstract}
${ }^{1}$ Shanghai Skin Disease Hospital, Shanghai, China ${ }^{2}$ National Microbiology Laboratory, Public Health Agency of Canada, Winnipeg, Canada
\end{abstract}

Correspondence to Dr Weiming Gu; weiming_gu2003@yahoo. com

\section{ABSTRACT}

Objective: In this study, we aimed to determine the performance characteristics of toluidine red unheated serum test on cerebrospinal fluids (CSF-TRUST) as compared to venereal disease research laboratory test on cerebrospinal fluids (CSF-VDRL) for laboratory the diagnosis of neurosyphilis.

Design: A cross-sectional study.

Setting: Sexually transmitted infections (STIs) clinics. Participants and methods: CSF and serum samples were collected from 824 individual STD clinic patients who have syphilis and are suspected to progress to neurosyphilis within a 9-month period. CSF-VDRL and CSF-TRUST were performed parallelly on the same day when collected. Treponema pallidum particle agglutination (TPPA) tests were also performed on the CSF and the serum samples, and biochemical analysis of the CSF samples was also performed.

Results: The overall agreement between CSF-TRUST and CSF-VDRL was $97.3 \%$. The reactive ratios of the CSF samples were $22.1 \%$ by CSF-TRUST and $24.8 \%$ by CSF-VDRL, respectively. All CSF-TRUST-reactive cases were reactive in the CSF-VDRL. Twenty-two samples with CSF-TRUST-negative were tested CSFVDRL-reactive with low titres (1:1 to $1: 4)$. Over $97 \%$ of the double-reactive CSF samples (CSF-VDRL and CSF-TRUST) had an identical titre or a titre within a two-fold difference. The agreement of CSF-TPPA and CSF-VDRL was $71.9 \%$. Similarly, the agreement of CSF-TPPA and CSF-TRUST was $69.2 \%$.

Conclusions: Our results revealed that CSF-TRUST could be used as an option for CSF examination in settings without CSF-VDRL in place.

Neurosyphilis can occur at any time in the course of syphilis, its symptoms and signs are usually non-specific. Some neurosyphilis cases remain asymptomatic. ${ }^{12}$ According to the $\mathrm{WHO}^{3}$ and the Center for Disease Control and Prevention (CDC USA), ${ }^{4}$ the venereal disease research laboratory test on cerebrospinal fluids (CSF-VDRL) is the method of choice for laboratory diagnosis of neurosyphilis. However, owing to the stability issues of VDRL antigens, no manufacturers in China

\section{ARTICLE SUMMARY}

Article focus

- Venereal disease research laboratory test (VDRL) is the method of choice for cerebrospinal fluid (CSF) examination in patients with suspected neurosyphilis. However, none of the commercial VDRL reagents has been approved by the China State Food and Drug Administration for use in CSF examination.

- Toluidine red unheated serum test on cerebrospinal fluids (CSF-TRUST)performs well, similarly to VDRL for syphilis serological tests.

- The purpose of this study is to evaluate whether CSF-TRUST can be used for neurosyphilis in settings lacking CSF-VDRL.

Key messages

- The overall agreement between CSF-VDRL and CSF-TRUST in 824 CSF samples from individual syphilis patients was $97.3 \%$.

- Over $97 \%$ of the double-reactive CSF samples (CSF-VDRL and CSF-TRUST) exhibited an identical titre or a titre within a two-fold difference.

- CSF-TRUST could be used as an alternative for CSF examination of neurosyphilis in settings without CSF-VDRL in place.

Strengths and limitations of this study

- There are currently no clear criteria for laboratory diagnosis of neurosyphilis. Therefore, this study can only determine the agreement of results for CSF-VDRL and CSF-TRUST.

- In our study, it was not possible to distinguish false-positive or false-negative for samples with inconsistent results of CSF-VDRL and CSF-TRUST.

are approved by the China State Food and Drug Administration Agency (SFDA) to supply the reagent commercially, which significantly hinders the correct and prompt diagnosis of neurosyphilis in Chinese hospital settings. ${ }^{5}$

TRUST is a modification of the VDRL procedure by labelling the antigen with toluidine red particles. ${ }^{6}$ TRUST is mostly used in serum tests for laboratory diagnosis of syphilis. The performance of TRUST for serum 
examination is similar to that of the rapid plasma regain (RPR). The TRUST reagents from commercial sources have been approved by the SFDA for syphilis diagnosis. Therefore, we are interested in determining whether TRUST can be used for CSF examination in suspected neurosyphilis cases. The objective of this study was to determine the performance characteristics of toluidine red unheated serum test on cerebrospinal fluids (CSF-TRUST) when compared to CSF-VDRL in CSF samples collected from 824 syphilis patients.

\section{MATERIALS AND METHODS}

\section{CSF and serum samples}

The criteria for diagnosis of syphilis and syphilis staging were those described by the National Center for STD Control, China CDC. ${ }^{7}$ The criteria for examining CSF of a syphilis patient in this study included one of the following (1) having neurological or psychiatric signs or symptoms or (2) exhibiting serofast state for more than 2 years or (3) request from patients because of anxiety of neurosyphilis. ${ }^{8}$ The diagnosis of neurosyphilis in our hospital followed the criteria set by the China CDC. ${ }^{7}$ Briefly, a patient is diagnosed having neurosyphilis when meeting the following three criteria: (1) syphilis history with signs and/or symptoms of neurological damages and serumreactive of non-treponemal-specific serological tests and reactive in treponemal-specific serological tests; (2) syphilis history with abnormal CSF examination results (white blood cell counts (WBC) $>10^{6} / 1$ and protein $>500 \mathrm{mg} / \mathrm{l}$ ) which cannot be explained otherwise and (3) syphilis history with CSF-VDRL-reactive.

CSF samples were collected using lumbar punctures from syphilis patients at the Shanghai Skin Disease Hospital $(\mathrm{SSDH})^{8}$ in Shanghai during the period of June 2009-February 2011. CSF samples containing visible particles, exhibiting pink colours or having visible traces of blood were excluded from the study. Totally, 824 CSF samples from syphilis patients (each sample from one individual patient) were examined in this study. CSF samples were tested on the same day they were collected. CSF white blood cell counts were done within $2 \mathrm{~h}$ after the lumbar puncture, and other tests were conducted within $4 \mathrm{~h}$.

Serum samples were also collected from individuals at the same time and tested for syphilis serology on same day.

The study was approved by the Shanghai Skin Disease Hospital Ethical Review Board. All participants provided informed consent in writing.

\section{CSF-VDRL, CSF-TRUST and CSF-TPPA}

The following practice was conducted to minimise potential bias from technicians. (1) Two technicians involved in the testing were blind from patients' IDs; (2) CSF-VDRL and CSF-TRUST tests were performed by a separate technician, respectively and (3) The laboratory supervisor who was blind from patients' IDs as well combined the two sets of testing results at the end of each day.
CSF samples were centrifuged prior to CSF-VDRL, CSF-TRUST and CSF-TPPA (Treponema pallidum particle agglutination) examinations which were performed parallelly. Semiquantitative tests of CSF-VDRL and CSF-TRUST were performed by a serially two-fold dilution of the CSF samples with $0.85 \%$ saline and tested by both methods. The highest dilution of a sample which gave a reactive result was then used to assign a titre value.

VDRL antigen with buffered saline was purchased from Becton, Dickinson and Company (Diagnostic Systems, Sparks, Maryland, USA) ${ }^{9}$ CSF-VDRL tests were performed according to the manufacturer's instruction ${ }^{9}$ with modifications as described by Larsen $e t a l .{ }^{10} \mathrm{~A}$ fresh antigen suspension was prepared within $2 \mathrm{~h}$ prior to VDRL reactions. A quantity of $10 \mu \mathrm{l}$ of the prepared antigen was incubated with $50 \mu \mathrm{l} \mathrm{CSF}$ samples for $8 \mathrm{~min}$ with rotation at $180 \mathrm{rpm}$ on a mechanical rotator.

CSF-TRUST tests were performed using the TRUST reagent from the Shanghai Rongsheng Biotech Co (Shanghai, China) according to the routine for serum testing, according to the manufacturer's instructions. ${ }^{11}$ The TRUST antigen suspension was mixed with CSF or diluted CSF samples on a white card; the formation of red clumps indicated a reactive result.

CSF-TPPA tests were conducted using the Serodia-TPPA reagent from Fujirebio Diagnostics Inc (Malven, Pennsylvania, USA). ${ }^{12}$ CSF sample and sensitised gelatin particles were added to a microtitre well and incubated at room temperature for $2 \mathrm{~h}$. Results were observed according to the manufacturer's instructions. ${ }^{12}$

\section{WBC counts and biochemical analysis of CSF}

CSF samples were subjected to WBC counts and biochemical tests (Hitachi 7020 and Backman-IMMAGE800) to determine levels of chloride, sugar, total protein and albumin. The normal ranges of these markers and predicted values in neurosyphilis were those previously described. ${ }^{13}$ Test results for samples with inconsistent CSF-VDRL and CSF-TRUST testing results were analysed.

\section{Serum-TRUST and serum-TPPA}

Serum-TRUST and serum-TPPA tests were conducted according to the manufacturer's instructions using the Shanghai Rongsheng Biotech Co TRUST reagent and the Serodia-TPPA reagent from Fujirebio Diagnostics Inc (Malven, Pennsylvania, USA), respectively. ${ }^{11}{ }^{12}$

\section{Statistical analysis}

The program SPSS V.13.0 from IBM (IBM Corp, New York, USA) was used according to the publisher's instruction. McNemar's test was used to compare paired proportions. ${ }^{14}$ A $p$ value of $<0.05$ was considered significant.

\section{RESULTS}

Agreement of results between CSF-VDRL, CSF-TRUST and CSF-TPPA tests

The 824 CSF samples were collected from 824 syphilis patients comprising 456 male and 368 female syphilis 
Table 1 Agreement of results between CSF-VDRL and CSF-TRUST

\begin{tabular}{|c|c|c|c|c|}
\hline \multirow{2}{*}{ Method } & & \multicolumn{2}{|c|}{ CSF-VDRL (n, \%) } & \multirow[b]{2}{*}{ Total } \\
\hline & & + & - & \\
\hline \multirow[t]{2}{*}{ CSF-TRUST (n, \%) } & + & $182(22.1)$ & $0(0)$ & $182(22.1)$ \\
\hline & - & $22(2.7)$ & $620(75.2)$ & $642(77.9)$ \\
\hline Total & & $204(24.8)$ & $620(75.2)$ & $824(100)$ \\
\hline
\end{tabular}

CSF-TRUST, toluidine red unheated serum test on cerebrospinal fluids; CSF-VDRL, venereal disease research laboratory test on

cerebrospinal fluids.

patients. The average age of the patients was 42.3 years, ranging from 10 to 82 years.

For undiluted CSF samples, an overall agreement of $97.3 \%$ was observed (95\% CI $96.2 \%$ to $98.4 \%$ ) between CSF-VDRL and CSF-TRUST (table 1). Among the 824 CSF samples, reactive proportions for VDRL and TRUST were $24.8 \%$ and $22.1 \%$, respectively. The reactive percents were significantly different between CSF-VDRL and CSF-TRUST $(p<0.001)$.

In addition, the agreement of CSF-TPPA and CSF-VDRL was $71.9 \%$. The reactive proportion was significantly higher for CSF-TPPA $(52.9 \%)$ than that for CSF-VDRL $(24.8 \%)(p<0.001$, table 2$)$. Similarly, the agreement of CSF-TPPA and CSF-TRUST was $69.2 \%$, and the reactive per cents for CSF-TPPA and CSF-TRUST were $52.9 \%$ and $22.1 \%$, respectively ( $\mathrm{p}<0.001$, table 2 ).

\section{Comparison of titres of CSF-VDRL and CSF-TRUST}

All 620 CSF samples with negative CSF-TRUST test results were also tested negative by the CSF-VDRL method. There were $22 \mathrm{CSF}$ samples which were CSF-VDRL-reactive but CSF-TRUST-negative (figure 1). There were $94 \mathrm{CSF}$ samples with identical titres, accounting for $51.6 \%$ of the CSF samples with both VDRL-reactive and TRUST-reactive (94/182, shadowed cells of figure 1). Moreover, there were $83 \mathrm{CSF}$ samples with only two-fold differences in the titres of VDRL and TRUST, accounting for $45.6 \%$ of the CSF samples with both VDRL-reactive and TRUST-reactive (83/182, open boxes of figure 1). Therefore, the per cent of CSF double-reactive samples $(\mathrm{n}=182)$ which had identical or a two-fold difference titres was $97.2 \%$.

Five CSF samples with double-reactive $(5 / 182,2.8 \%)$ displayed significantly higher VDRL titres than TRUST (four-fold difference), whereas no CSF samples had a significantly higher TRUST titres than that of CSF-VDRL.

Analysis of samples with inconsistent results of CSF-VDRL and CSF-TRUST

All the CSF-TRUST-reactive samples in this study were also CSF-VDRL-reactive $(\mathrm{n}=182)$. On the other hand, there were 22 CSF-VDRL-reactive samples $(2.7 \%)$ which tested negative by the CSF-TRUST method. These $22 \mathrm{CSF}$ samples were CSF-TPPA-reactive. The serum antibody titres of these patients were in the range $1: 4$ to $1: 64$ for serum-TRUST and had a titre of higher than 1:640 for serum-TPPA.

The 22 patients comprised neurosyphilis $(\mathrm{n}=16)$, primary syphilis $(n=1)$, secondary syphilis $(n=3)$ and latent syphilis $(\mathrm{n}=2)$ (table 3). Three cases had a CSF-VDRL titre of 1:2 or $1: 4$, whereas the rest 19 cases exhibited CSF-VDRL-reactive in undiluted CSF.

WBC and biochemical analysis were performed on the 22 CSF samples with inconsistent CSF-VDRL and CSF-TRUST results (table 3). Seven of the 22 CSF samples showed a remarkable increase in WBC counts $\left(>8 \times 10^{6} / 1\right)$, which comprised four neurosyphilis cases, one latent syphilis and two secondary syphilis patients. Eight CSF samples had slightly lower chloride concentration than the normal ranges, comprising seven samples from neurosyphilis and one from latent syphilis cases. Two samples exhibited abnormal CSF sugar concentrations, which were from neurosyphilis patients. Four CSF samples from one latent, one primary and two neurosyphilis cases had slightly higher levels of total proteins than the normal ranges. Twelve CSF samples exhibited increased albumin

Table 2 Agreement of results between CSF-VDRL and CSF-TPPA or between CSF-TRUST and CSF-TPPA

\begin{tabular}{|c|c|c|c|c|c|c|c|}
\hline \multirow{2}{*}{ Method } & & \multicolumn{3}{|c|}{ CSF-VDRL (n, \%) } & \multicolumn{3}{|c|}{ CSF-TRUST (n, \%) } \\
\hline & & + & - & Subtotal & + & - & Subtotal \\
\hline \multirow[t]{2}{*}{ CSF-TPPA (n, \%) } & + & $204(24.8)$ & $232(28.2)$ & $436(52.9)$ & $182(22.1)$ & $254(30.8)$ & $436(52.9)$ \\
\hline & - & $0(0.0)$ & $388(47.1)$ & $388(47.1)$ & $0(0.0)$ & $388(47.1)$ & $388(47.1)$ \\
\hline Subtotal & & $204(24.8)$ & $620(75.2)$ & 824 & $182(22.1)$ & $642(77.9)$ & 824 \\
\hline p Value & & & $<0.001^{\star}$ & & $<0.00$ & & \\
\hline
\end{tabular}

${ }^{*}$ Comparison of reactive per cents for CSF-VDRL and CSF-TPPA.

†Comparison of reactive per cents for CSF-TRUST and CSF-TPPA.

CSF-TRUST, toluidine red unheated serum test on cerebrospinal fluids; CSF-VDRL, venereal disease research laboratory test on cerebrospinal fluids; CSF-TPPA, treponema palladium particle agglutination test on cerebrospinal fluids. 


\begin{tabular}{|c|c|c|c|c|c|c|c|c|c|}
\hline \multirow{2}{*}{ Method } & & \multicolumn{8}{|c|}{ CSF-TRUST (N) } \\
\hline & Titer & - & $1: 1$ & $1: 2$ & $1: 4$ & $1: 8$ & $1: 16$ & $\geq 1: 32$ & Subtotal \\
\hline \multirow{8}{*}{$\begin{array}{l}\text { CSF-VDRL } \\
\text { (N) }\end{array}$} & - & 620 & 0 & & & & & & 620 \\
\hline & 1:1 & 19 & 28 & 2 & & & & & 49 \\
\hline & $1: 2$ & -1 & 25 & 24 & 2 & & & & 53 \\
\hline & 1:4 & 1 & 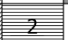 & 19 & 18 & 1 & & & 41 \\
\hline & $1: 8$ & & & 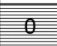 & 20 & 16 & 1 & & 37 \\
\hline & 1:16 & & & & 2 & 10 & 7 & 0 & 19 \\
\hline & $\geq 1: 32$ & & & & & 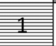 & 3 & 1 & 5 \\
\hline & Subtotal & 642 & 55 & 45 & 42 & 28 & 11 & 1 & 824 \\
\hline
\end{tabular}

Figure 1 Semiquantitative analysis of CSF-VDRL and CSF-TRUST. Shadowed boxes indicate samples with identical titres of CSF-VDRL and CSF-TRUST. Open boxes indicate samples having two-fold dilution difference between CSF-VDRL and CSF-TRUST. Upward diagonals indicate CSF-VDRL-reactive, but CSF-TRUST non-reactive $(n=22)$. Horizontal strike boxes indicate that CSF-VDRL titres are significantly higher than CSF-TRUST titres $(n=5)$. -, Negative; CSF-TRUST, toluidine red unheated serum test on cerebrospinal fluids; CSF-VDRL, venereal disease research laboratory test on cerebrospinal fluids.

concentrations, comprising one latent syphilis, three secondary syphilis and eight neurosyphilis cases.

\section{DISCUSSION}

A combination of biochemical, WBC examination and VDRL analysis of CSF sample is the recommended method for the laboratory diagnosis of neurosyphilis. ${ }^{1-3} 15$ For patients who are neurologically asymptomatic, assessment of the efficacy of neurosyphilis treatment relies on normalisation of CSF-VDRL measures and other CSF measures such as WBC counts, concentrations of CSF total protein, chloride, sugar and albumin. ${ }^{16-18}$ However, CSF-VDRL is a complicated process, the antigen suspension needs to be freshly prepared and used within $2 \mathrm{~h}$. As pointed out by Parham et al, ${ }^{19}$ the reproducibility of VDRL method is reagent and operator dependent. In their study, the agreement of CSF-VDRL results between two top CDC laboratories was $87 \% .{ }^{19}$ The situation is even more challenging in China as there are no commercial VDRL reagents approved by the SFDA for CSF-VDRL examination. On the other hand, the TRUST method is a modified version of the VDRL test, ${ }^{6}$ the toluidine red particle-labelled antigen is stable at room temperature ( $\mathrm{Gu}$ et al, unpublished data), can be performed as a simple agglutination test; and there are multiple commercial TRUST reagents approved by SFDA for use in serum examination in the laboratory diagnosis of syphilis.

We investigated the potential use of the TRUST method for the laboratory diagnosis of neurophilis with CSF samples collected from 824 syphilis patients. The agreement of CSF-VDRL and CSF-TRUST was $97.3 \%$, and $97.2 \%$ of the reactive CSF samples had similar titres (within two-fold dilutions) determined by the two methods. Moreover, the agreements between CSF-TPPA and CSF-VDRL $(71.9 \%)$ and between CSF-TPPA and CSF-TRUST $(69.2 \%)$ were similar. This study suggests that CSF-TRUST could be an option in CSF-VDRL unavailable settings.

Pettit et al evaluated the performance of the TRUST method against the VDRL method on serum samples for the diagnosis of syphilis. Their results indicated a $99.7 \%$ agreement between the two methods. ${ }^{6}$ Recent publication by Jiang et al revealed the potential use of CSF-TRUST in neurosyphilis diagnosis by examining archived CSF samples from neurosyphilis. ${ }^{20}$

Discrepancy between CSF-VDRL and CSF-TRUST was observed in this study. First, $2.8 \%$ of double-reactive samples (5/182) showed significantly higher VDRL titres than TRUST (four-fold difference). Second, the 22 CSF-TRUST non-reactive samples had low titres of CSF-VDRL, and a portion of these samples also had abnormal CSF biochemical parameters such as WBC counts, concentrations of sugar, chloride, total protein and albumin. Third, six cases were diagnosed as

Table 3 Biochemical analysis of 22 CSF samples with VDRL-reactive, but TRUST-non-reactive

\begin{tabular}{|c|c|c|c|c|c|c|c|}
\hline $\begin{array}{l}\text { Clinical } \\
\text { diagnosis* }\end{array}$ & $\begin{array}{l}\text { Number } \\
\text { of cases }\end{array}$ & VDRL titres & $\begin{array}{l}\text { WBC } \\
\text { Range (n } \\
\text { abnormal)† }\end{array}$ & $\begin{array}{l}\text { Chloride } \\
\text { Range (n } \\
\text { abnormal)† }\end{array}$ & $\begin{array}{l}\text { Sugar } \\
\text { Range (n } \\
\text { abnormal)† }\end{array}$ & $\begin{array}{l}\text { Total protein } \\
\text { Range (n } \\
\text { abnormal)† }\end{array}$ & $\begin{array}{l}\text { CSF-albumin } \\
\text { Range ( } \mathrm{n} \\
\text { abnormal)† }\end{array}$ \\
\hline Neurosyphilis & 16 & $1: 1-1: 4$ & $0-18.8$ (4) & $115-126(7)$ & $3.57-6.54(2)$ & $0.19-1.09$ (2) & $0.14-0.87(8)$ \\
\hline Primary & 1 & $1: 1$ & $3.0(0)$ & $123.0(0)$ & $3.34(0)$ & $0.36(1)$ & $0.23(0)$ \\
\hline Secondary & 3 & $1: 1$ & $2-104.5(2)$ & $121-122(0)$ & $2.86-3.81(0)$ & $0.32-0.44(0)$ & $0.28-0.39$ (3) \\
\hline Latent & 2 & $1: 1$ & $0-14.5$ (1) & 118-122 (1) & $3.45-4.28(0)$ & $0.28-0.33(1)$ & $0.22-0.25(1)$ \\
\hline Total/range & 22 & $1: 1-1: 4$ & $0-104.5$ (7) & $115-126(8)$ & $2.86-6.54(2)$ & $0.19-1.09$ (4) & $0.14-0.87(12)$ \\
\hline
\end{tabular}

*Diagnosis was made by physician's presumptive diagnosis, pending laboratory tests.

†Number of samples with abnormal values.

CSF WBC: normal range $0-10^{6} /$.

CSF chloride: normal range $122-132 \mathrm{mmol} / \mathrm{l}$.

CSF sugar: normal range $2.5-4.5 \mathrm{mmol} / \mathrm{l}$.

CSF total protein: normal range $0.15-0.45 \mathrm{mg} / \mathrm{l}$.

CSF-albumin: normal range $0.139-0.246 \mathrm{mg} / \mathrm{l}$.

CSF-TRUST, toluidine red unheated serum test on cerebrospinal fluids; CSF-VDRL, venereal disease research laboratory test on cerebrospinal fluids; WBC, white blood cell count. 
'non-neurosyphilis' exhibited CSF-VDRL-reactive but CSF-TRUST non-reactive, and these cases also showed abnormal values of CSF biochemical markers. This suggests that CSF-TRUST is less sensitive than CSF-VDRL when the antibody titres are low. Therefore, while CSF-TRUST-reactive could be diagnostic of neurosyphilis, CSF-TRUST non-reactive patients should be further investigated to rule out neurosyphilis.

Other earlier studies have indicated that negative CSF treponemal-specific tests may not exclude the diagnosis of neurosyphilis. ${ }^{21}$ We also examined the agreement of a CSF treponemal-specific test CSF-TPPA to CSF-VDRL and CSF-TRUST. The low per cents of agreement $(\sim 70 \%)$ suggest that CSF-TPPA is not a good method for laboratory diagnosis of neurosyphilis. However, we did not examine the sensitivity and specificity of CSF-TPPA for neurosyphilis in our study.

The use of CSF-RPR in neurosyphilis diagnosis have also been evaluated by others. ${ }^{19} 2223$ Marra $e t a l^{22}$ have recently evaluated CSF-RPR performance on 149 syphilis patients and found that the sensitivities of CSF-RPR for laboratorydiagnosed neurosyphilis was $56.4 \%$ (serum RPR method) or $59 \%$ (CSF RPR method) compared to $71.8 \%$ of CSF-VDRL. ${ }^{22}$ CSF-RPR also showed lower titres than CSF-VDRL. ${ }^{22}$ Study by Castro et at $t^{23}$ revealed similar sensitivities for CSF-RPR (75\%) and CSF-VDRL (70.8\%), and that both methods had specificities of over $99 \%$. The discrepancy of the results from these two studies may, at least partly, be owing to the choice of negative and positive controls. ${ }^{21}$

The use of CSF-TRUST for the evaluation of treatment effects of neurosyphilis remains unclear. It was reported that the normalisation time (median months) of CSF-VDRL and serum RPR was 3.9 and 4.4 months after treatments, respectively. ${ }^{18}$ Syphilis stages and HIV-coinfections are some of the factors influencing the normalisation time.

Our study has the limitations of defining neurosyphilis status in the study subjects because of the lack of clinical data. It is difficult to interpret the performance of CSF-TRUST in terms of its specificity, sensitivity and predictive values in sexually transmitted infections (STIs) clinic settings. It seems that CSF-TRUST and CSF-VDRL are congruence in most cases, but CSF-TRUST is less sensitive than CSF-VDRL. Nevertheless, our results suggested that CSF-TRUST may be used as an alternative for laboratory diagnosis of neurosyphilis in clinical settings with CSF-VDRL unavailable.

Acknowledgements We specially thank Raymond SW Tsang (National Microbiology Laboratory, Public Health Agency of Canada) and Mingmin Liao (Vaccine and Infectious Disease Organization, University of Saskatchewan, Canada) for their critiques and editing of this manuscript. We thank Yue Chen (Epidemiology and Community Medicine, University of Ottawa) for reviewing the statistical analysis.

Contributors WG was the principal investigator and lead writer. YY was the co-investigator. LW and SY were technical personnel at SSDH. LKN is a collaborator from NML Canada. YY, LW and SY significantly contributed to acquisition of data. All authors helped plan the study, interpret data and critically revise successive drafts of the manuscript.
Funding This work was supported by the Shanghai Nature Science Fund, Shanghai, China (\#02ZB14084 and \#09DZ1907104).

Competing interests None.

Patient consent Obtained.

Ethics approval Ethics board of the Shanghai Skin Disease Hospital, Shanghai, China.

Provenance and peer review Not commissioned; externally peer reviewed.

Data sharing statement There are no additional data available.

\section{REFERENCES}

1. Christina MM. Update on neurosyphilis. Curr Infect Dis Rep 2009;11:127-34.

2. Patrick RM, Ellen JB, James HJ, et al. eds. Treponema and other human host-associated spirochetes. In: Manual of clinical microbiology. 9th edn. Washington DC: American Society for Microbiology, 2007:987-1003.

3. Department of Reproductive Health and Research, World Health Organization. The global elimination of congenital syphilis: rationale and strategy for action. Geneva: WHO, 2007.

4. Ronald LM, Christine GC, Teresa FR, et al. Sexually transmitted diseases treatment guidelines. MMWR Morb Mortal Wkly Rep 2010;59:27-8.

5. http://www.sfda.gov.cn (accessed 28 Aug 2012).

6. Pettit DE, Larsen SA, Harbec PS, et al. Toluidine red unheated serum test, a nontreponemal test for syphilis. J Clin Microbiol 1983:18:1141-5.

7. Wang Q, Zhang G, eds. Guidelines for diagnosis and treatment of sexually transmitted diseases. National Center for STD Control, China CDC. Shanghai, China: Shanghai scientific \& Technical Publishers. 2007.

8. Zhou P, Gu X, Lu H, et al. Re-evaluation of serological criteria for early syphilis treatment efficacy: progression to neurosyphilis despite therapy. Sex Transm Infect 2012;88:342-5.

9. Becton, Dickinson Co., Maryland, USA. http://www.bd.com/ (accessed 28 Aug 2012)

10. Larsen SA, Pope V, Johnson RE, et al. A manual of tests for syphilis. 9th edn. Washington, DC: American Public Health Association, 1998.

11. Shanghai Rongsheng Biotech Co., Ltd. Shanghai, China. http:// www.rsbio.com (accessed 28 Aug 2012).

12. Diagnostics, Inc. Japan. http://www.fdi.com/index.html (accessed 28 Aug 2012).

13. Ye YW. National guide to clinical laboratory procedures. 3rd edn. Medical administration department of Ministry of Public Health, China. Nanjing, China: Southeast University Press, 2 Nov, 2006.

14. Hoffman JI. The incorrect use of Chi-square analysis for paired data. Clin Exp Immunol 1976:24:227-9.

15. French $\mathrm{P}$, Gomberg M, Janier M, et al. 2008 European guidelines on the management of syphilis. Int J STD \& AIDS 2009;20:300-9.

16. Dijkstra JW. Asymptomatic neurosyphilis. Int J Dermatol 1983;22:581-9.

17. Marra CM, Maxwell CL, Tantalo LC, et al. Normalization of serum rapid plasma reagin titer predicts normalization of cerebrospinal fluid and clinical abnormalities after treatment of neurosyphilis. Clin Infect Dis 2008;47:893-9.

18. Marra CM, Maxwell CL, Tantalo L, et al. Normalization of cerebrospinal fluid abnormalities after neurosyphilis therapy: does HIV status matter? Clin Infect Dis 2004;38:1001-6.

19. Parham CE, Pettit DE, Larsen SA, et al. Interlaboratory comparison of the toluidine red unheated serum test antigen preparation. $J$ Clin Microbiol 1984;20:434-7.

20. Jiang $\mathrm{Y}, \mathrm{Chen} \mathrm{X}, \mathrm{Ma} \mathrm{X}$, et al. The usefulness of toluidine red unheated serum test in the diagnosis of HIV-negative neurosyphilis. Sex Transm Dis 2011;38:244-5.

21. Harding AS, Ghanem KG. The performance of cerebrospinal fluid treponemal-specific antibody tests in neurosyphilis: a systematic review. Sex Transm Dis 2012;39:291-7.

22. Marra CM, Tantalo LC, Maxwell CL, et al. The rapid plasma reagin test cannot replace the venereal disease research laboratory test for neurosyphilis diagnosis. Sex Transm Dis 2012;39:453-7.

23. Castro R, Prieto ES, da Luz Martins Pereira F. Nontreponemal tests in the diagnosis of neurosyphilis: an evaluation of the venereal disease research laboratory (VDRL) and the rapid plasma reagin (RPR) tests. J Clin Lab Anal 2008;22:257-61. 